\title{
ANÁLISIS DE OPORTUNIDADES PARA EL DESARROLLO DE ACTIVIDADES DE TURISMO ALTERNATIVO EN LA REGIÓN NORTE DE BAJA CALIFORNIA SUR (MÉXICO)
}

\section{ANALYSIS OF OPPORTUNITIES FOR THE DEVELOPMENT OF ALTERNATIVE TOURISM ACTIVITIES IN THE NORTH REGION OF BAJA CALIFORNIA SUR (MÉXICO)}

Emmanuel Picasso Salazar ${ }^{1}$

Francisco Isaías Ruiz Ceseña² Alberto Francisco Torres García ${ }^{3}$

1. Licenciado en Turismo Alternativo, Estudiante de posgrado Maestría en Administración Estratégica en el Departamento Académico de Economía, Universidad Autónoma de Baja California Sur (México). E-mail: picasso.emmanuel@hotmail.com

2. Maestro en Economía Aplicada con estudios en el área de Economía Aplicada y Desarrollo Económico. Es profesor-investigador del Departamento Académico de Economía en la Universidad Autónoma de Baja California Sur (México). E-mail: isaias@uabcs.mx

3. Doctor en Relaciones Transpacíficas con estudios en Economía Internacional y Competitividad Internacional. Es profesor-investigador del Departamento Académico de Economía en la Universidad Autónoma de Baja California Sur (México). E-mail: atorresg@uabcs.mx

\section{Citación sugerida:}

Picasso Salazar, E., Ruiz Ceseña, F.I. y Torres García, A.F. (2018). Análisis de oportunidades para el desarrollo de actividades de turismo alternativo en la región norte de Baja California Sur (México). $3 C$ Empresa: investigación y pensamiento crítico, 7(1), 32-48. DOI: $<$ http://dx.doi.org/10.17993/3cemp.2018.070133.32-48/>. 


\section{RESUMEN}

Baja California Sur (BCS) encuentra en el turismo su actividad económica por excelencia al contar con uno de los destinos turísticos más importantes a nivel nacional e internacional situado en la parte sur del estado, nos referimos a Los Cabos. Sin embargo, dadas las condiciones geográficas de la entidad resulta difícil que el norte se vea beneficiado por la actividad turística desarrollada en el destino recién mencionado. El norte de BCS cuenta con la presencia de recursos naturales altamente potenciales para el desarrollo de actividades turísticas dirigidas al turismo de naturaleza. En este sentido, resulta pertinente llevar a cabo un estudio que exponga las oportunidades en la zona en juicio para el desarrollo de actividades de turismo basado en los atractivos naturales, ocasionando que el área delimitada obtenga un beneficio económico en las comunidades que no participan en la generación de divisas por el turismo llevado a cabo en el sur de BCS. En la presente investigación se enlistan estudios que fueron enfocados en el norte del estado. Frente al hecho que éstos se limitaron únicamente a aprobar o descartar el potencial turístico del área, el presente documento busca analizar las investigaciones mencionadas y a partir de sus resultados obtenidos, estudio de campo y exploración del área obtener como resultado la determinación detallada de cuáles son las actividades de turismo alternativo aptas a desarrollarse en la zona norte. Se concluye que la armonización de ambas partes del territorio y las distintas modalidades de turismo permiten que todo el estado sea generador de divisas.

\section{ABSTRACT}

Baja California Sur (BCS) is one of the most important tourist destinations in the southern part of the state. We are talking about Los Cabos. However, given the geographical conditions of the entity it is difficult for the north to benefit from the tourism activity developed in the aforementioned destination. The north of BCS has the presence of highly potential natural resources for the development of tourist activities directed to nature tourism. In this sense, it is pertinent to carry out a study that exposes the opportunities in the trial zone for the development of tourism activities based on the natural attractions, thus leading to the delimited area to obtain an economic benefit in communities that do not participate in The generation of foreign exchange by the tourism carried out in the south of BCS. In the present investigation are listed studies that were focused in the north of the state. Faced with the fact that these were limited only to approve or discard the tourist potential of the area, this document seeks to analyze the mentioned research and based on their results, field study and exploration of the area to obtain as a result the detailed determination of which are the Alternative tourism activities suitable for development in the north. It is concluded that the harmonization of both parts of the territory and the different forms of tourism allow the whole state to generate foreign exchange. 


\section{PALABRAS CLAVE}

Turismo Alternativo, Oportunidades, Desarrollo Económico, Desarrollo Local, Baja California Sur.

\section{KEY WORDS}

Alternative Tourism, Opportunities, Economic Development, Local Development, Baja California Sur. 


\section{INTRODUCCIÓN}

La Organización Mundial del Turismo (OMT) afirma que a lo largo de las décadas el turismo ha tenido un desarrollo que lo ha convertido en uno de los sectores de mayor crecimiento económico en el mundo. El turismo está relacionado con el desarrollo y abarca un número creciente de nuevos destinos, lo que lo ha convertido en un motor económico clave para el crecimiento socioeconómico (OMT, 2015). La extensión que ha tenido ha dejado beneficios económicos y de empleo en sectores relacionados con el mismo, desde la construcción hasta las telecomunicaciones (OMT, 2015). El diagnóstico del sector turismo en México (López y González, 2012) señala que sin importar la fuerte crisis que ha afectado al mundo ni el problema de inseguridad, México siguió posicionado como uno de los 10 destinos turísticos más visitados. Sin embargo, estudios realizados durante el 2013 afirman que México pasó del décimo al decimotercer país que recibió más turistas (IMCO, 2013) y en 2015, de acuerdo con el Barómetro del Turismo Mundial ocupó el decimoquinto puesto (Ruiz, 2016).

Para el año 2010 México recibió más de 22 millones de turistas extranjeros y más de 51 millones de visitantes de zonas fronterizas, dejando una derrama económica de 11 mil 760 millones de dólares y es considerado el primer destino turístico de los estadounidenses. México es el primero en el mundo en términos del número de visitas de cruceros, teniendo más de 6 millones de pasajeros (López y González, 2012). En 2010, el sector turismo continuó siendo un pilar de la economía en México: representó el 9\% del PIB generando 2.5 millones de empleos directos. De acuerdo con la OMT (2015), el turismo dejó un total de 13.9 miles de millones de dólares, cifra que representó una variación porcentual del 9.5\% con respecto al año anterior. El Instituto Nacional de Estadística y Geografía (INEGI), afirma que "durante el primer trimestre de 2015 el Indicador Trimestral del PIB turístico creció 1.0 por ciento con cifras desestacionalizadas frente al trimestre inmediato anterior" (Informador, 2015).

Datos que presenta Ruiz, (2016) y citando al Banco de México indican que en 2016 percibió un total de 2 millones de personas, datos que se traducen en un total de $\$ 1,389$ millones de dólares. En este orden de ideas, podemos concluir que México es un destino competitivo que dentro de sus actividades económicas sobresale el sector terciario. Dicha actividad, como se ha manejado en los datos históricos, ha sido suficiente para estimular sus ingreso. Es entonces importante impulsar nuevos destinos y actividades turísticas que sean acordes a las condiciones que presenten.

Baja California Sur encuentra en el turismo su principal actividad económica. Dicha actividad ha aumentado de manera considerable los últimos 25 años. De acuerdo con el Sistema Integral de Información de Mercados Turísticos (SIIMT, 2016) BCS ocupa el lugar 21 entre los estados en lo que a llegadas de turistas se refiere. En el año 2012, el turismo representó el $44 \%$ de su PIB y la principal fuente de ingresos en donde se emplearon a más de 37,000 sudcalifornianos de manera directa y 148,000 de manera indirecta (Reachi, 2012). Actualmente, el turismo cubre el $75 \%$ de su PIB (Mendoza, 2016). En este sentido, más que un servicio representa un pilar económico que beneficia a sudcalifornia. Se debe 
mencionar que el destino turístico de Los Cabos, por sí solo genera el 96\% de todo el PIB estatal (Ortiz, 2016). Esto lo podemos respaldar con lo que afirman Ibáñez, Cruz y Juárez (2016) "[...] alberga $73 \%$ de la infraestructura de hospedaje y cuenta con $65 \%$ de la afluencia de visitantes".

Los Cabos es considerado el segundo destino turístico más atractivo de México, después de Cancún, Quintana Roo. Ambos pertenecen al programa de Centros Integralmente Planeados (CIP) diseñado durante la época de los setentas para impulsar al turismo y su desarrollo en puntos estratégicos para que esos lugares constituyeran ventajas en cuanto a recursos regionales. Dicha estrategia fortaleció al turismo (Cruz, et. al. 2015), principalmente a la modalidad de turismo de sol y playa. De acuerdo con Cruz, (2015) "a partir de su creación hasta el año 2011 la tasa de crecimiento media anual de la afluencia turística total al CIP Los Cabos fue de 70\%, en cuanto a turismo nacional e internacional estos crecieron en una tasa media anual de 45 y $89 \%$, respectivamente".

Podemos concluir que en BCS, el desarrollo dentro del sector terciario se ha concentrado en el apoyo directo a las actividades del turismo masivo en la parte sur, lo que ha significado una derrama económica considerable. Sin embargo la parte norte no se ha desarrollado con el ímpetu que ha caracterizado el sur del mismo. Pretendemos resaltar mediante estudios recientes e innovadores las opciones de desarrollo turístico para la parte norte, que si bien no cuenta con infraestructura para albergar a una cantidad masiva de turistas, cuenta con riquezas naturales y atractivas que le permitirían aprovecharlas para actividades de turismo acorde a sus potencialidades.

\section{REVISIÓN LITERARIA}

Los principales puntos a teorizar dentro de este apartado nos permitirán determinar estrategias vinculadas al turismo presentado. Al tratarse de una actividad dependiente de los recursos naturales que resulten atractivos para el turista, es necesario presentar distintas definiciones del concepto "recurso turístico natural", lo que nos permitirá contar con una visión más clara de los recursos en los cuales nos hemos de enfocar. Como segundo punto, y una vez comprendidas las definiciones mencionadas, expondremos el término de turismo alternativo bajo el sustento de distintos autores, esto al ser la actividad deseada a desarrollar dentro de los recursos y sus atractivos turísticos naturales. Finalmente, presentaremos y nos enfocaremos en aquellos estudios que se llevaron a cabo estrictamente en la parte norte de BCS y que compartan intenciones de que el turismo alternativo sea una vía de desarrollo para la zona.

\subsection{RECURSO TURÍSTICO NATURAL}

El aprovechamiento de los recursos naturales tiene distintas vertientes. Para Boullón, (2006), uno de los más claros ejemplos la comparación entre el sector industrial y el sector turístico, ya que en la industria la materia prima es extraída para ser transformada, caso 
contrario del turismo, donde los atractivos deben permanecer intactos para el aprovechamiento de los mismos en un futuro. Bajo esta dinámica se definirá a continuación el término recursos naturales apegándolo a lo referente en materia turística.

De acuerdo con Goeldner y Ritchie (2011), se trata del conjunto de recursos naturales con el medio ambiente que lo rodea dentro de un área y que se encuentran disponible para el uso y disfrute de los visitantes, entre ellos los autores destacan el aire, clima, terreno, flora, fauna, playas y la belleza natural presente.

Boullón (2006) los define como todas las expresiones de la naturaleza que tienen interés turístico, tales como montañas, lagos, lagunas, esteros, lugares de observación de flora y fauna, planicies, entre otros. Además, sustenta que "se entremezclan con otro tipo de atractivos, como sitios arqueológicos, los grupos étnicos, las represas o algunas fiestas religiosas o profanas" (Boullón, 2006).

Tisdell (2003), coincide con Boullón (2006) al afirmar que los recursos naturales turísticos se presentan de manera combinada entre lo existente naturalmente y atractivos que generan una mayor atracción para el turista. Sin embargo, no comparte la idea de los autores anteriores en función de que es un conjunto de aspectos naturales para el disfrute del turista, pues sostiene que "hay pocos recursos naturales que son solamente recursos turísticos y normalmente esto debe tomarse en cuenta evaluando los recursos naturales usados para fines turísticos" (Tisdell, 2003), aclarando de esta manera que un inventario de recursos turísticos no es listado de recursos naturales, sino que es necesario discernir entre cuáles realmente son atractivos para el turista.

Covarrubias, (2015) afirma que los recursos naturales son aquellos que no han sufrido alteración ni modificaciones, de esta manera resultan atractivos para ser visitados creando así en el turista un motivante de desplazamiento. Es decir, el autor denomina como recurso natural turístico únicamente a aquellos en los que el hombre no ha tenido colaboración.

Por su parte, para Bertoni (2005), los recursos naturales turísticos originalmente son factores de atracción que tienen la característica de estar fijados naturalmente en el espacio y promover flujos de personas con finalidades turístico-recreativas.

En este orden de ideas y con base en los autores citados, podemos definir claramente a los recursos naturales como: aquellos existentes dentro de un espacio que no ha sido creado ni alterado por el hombre. No obstante, al tratarse de atractivos turísticos naturales, como bien lo propone Tisdell (2003), es necesario indagar más allá de recursos de los cuales la naturaleza nos dotó, sino conocer realmente cuáles pueden del disfrute del turista, generando en los recursos, como lo sustenta Bertoni (2005) un flujo de turistas que son atraídos por los recursos. Esto nos lleva a coincidir con Arrrillaga 2001, en Landa (2015), quien sostiene que los atractivos turísticos son el motivante de desplazamiento por el cual el viajero realiza visitas a otros sitios.

\subsection{TURISMO ALTERNATIVO}

La Secretaría de Turismo (SECTUR), como principal órgano en dicha materia a nivel nacional ha conceptualizado el término turismo alternativo, sin embargo, esto no exenta a que 
diversos autores tomen una definición distinta a la presentada por la secretaría. Es por ello que definirlo como tal debe considerar distintos conceptos propuestos por los investigadores relacionados con el tema. Por ello, para juicio del presente trabajo se tomarán como base definiciones de diversos autores mediante una recopilación de distintas ópticas referentes al tema que nos ocupa.

A continuación, se presenta una recopilación de definiciones que se han generado a partir del turismo alternativo en el que sobresalen organizaciones, cuerpos de investigación e investigadores individuales.

Secretaría de Turismo: El turismo alternativo es un segmento nuevo del turismo global que está cobrando cada vez mayor importancia. Este turismo trata de organizar su viaje hacia lugares desconocidos, conforme a sus necesidades y tiempos; busca descubrir sitios alejados del turismo de masas en ambientes naturales y que estimulen su desarrollo personal. A diferencia del turismo masivo, el turismo alternativo está dispuesto a pagar precios altos por la existencia de atractivos únicos, ya sean naturales o culturales (SECTUR, 2004).

Secretaría de Turismo \& Centro de Estudios Superiores del Turismo: "Un concepto que agrupa actividades turístico-recreativas que las personas realizan durante sus viajes y estancias, las cuales a pesar de diversa temática, convergen en que el atractivo focal en torno al cual giran es la naturaleza" (Elizondo, Cárdenas \& Teresa, 2007).

Casal Zamorano: Un conjunto de vivencias y experiencias únicas, irrepetibles, personales que se dan en un entorno de calidad; entendida ésta como la calidad en el tiempo libre del turista, en un entorno de calidad geográfica y social, que permite al turista percibir sus relaciones de una manera diferente con el entorno geográfico y cultural, con los otros turistas y sus anfitriones, dentro de los parámetros del desarrollo humano sustentable que prevé el desarrollo y crecimiento económico, la equidad social y la sustentabilidad ambiental (Zamorano, 2008).

Bertoni (2005): Actividades turísticas caracterizadas por el uso de los recursos naturales, preferentemente, en un estado de escasa intervención humana (Bertoni, 2005).

Acerenza (1995) en Quesada Castro (2007): ...Toda una gama de alternativas de viajes... que tienden a apartarse de las características que presenta el turismo masivo... incluyen, entre otras al turismo cultural, al turismo de aventura, al turismo rural y al turismo ecológico o ecoturismo... Las personas que practican el turismo alternativo... desean conocer y tener un contacto más estrecho con los pueblos y sus manifestaciones culturales, experimentar nuevas emociones o realizar alguna actividad fuera de lo común, lejos de la civilización, conocer y disfrutar la práctica de las labores del campo, o simplemente aprovechar los beneficios de la naturaleza (Quesada, 2007).

En función de las definiciones mencionadas, podemos concluir que: se entiende por turismo alternativo aquellas actividades que invitan a los practicantes a disfrutar y respetar los sitios visitados, siendo éstos escenarios únicos, lo que les lleva a vivir una experiencia distinta en la que no sólo son espectadores, sino están involucrados en ella retomando lo que sostiene la SECTUR, dividiendo este tipo de turismo en tres pilares, "Ecoturismo, Turismo de Aventura y Turismo Rural" (SECTUR, 2004). 


\subsection{INVESTIGACIONES ENFOCADAS EN EL ÁREA DE ESTUDIO}

Las investigaciones enfocadas en el norte de BCS abordan el tema desde cómo el turismo alternativo presenta una opción de desarrollo para la zona. Sin embargo, al hecho de que los estudios se encuentran de manera dispersa, resulta difícil aterrizar sobre oportunidades existentes en el área. Además, cabe mencionar que en los estudios en mención su aporte concluyó en comprobar o rechazar si los sitios estudiados cuentan con potencial turístico alternativo, sin delimitar detalladamente qué actividades de la modalidad mencionada son posibles de llevarse a cabo en el área de estudio. A continuación se enlistan los estudios a ser analizados.

Evaluación del potencial turístico rural como línea de acción para el desarrollo regional sustentable en los oasis de San Isidro y La Purísima, Baja California Sur: Estudio que respalda resultados en una metodología aplicada y desarrollada para el estudio de una zona en específico, utiliza conceptos de distintas corrientes teóricas y de aplicación adaptadas al Turismo Rural (Arce \& Ruiz, 2016).

Desarrollo de una metodología determinar el potencial del turismo rural en oasis sudcalifornianos: Proyecto de investigación orientado en realizar un análisis de las ventajas comparativas y competitivas de las localidades asentadas en los principales Oasis ubicados en BCS. El estudio se basa en dos metodologías formulando una, la primera involucrada analiza variables comparativas naturales para determinar su potencialidad (Modelo Calgary), la segunda metodología involucrada analiza variables competitivas (Monitor WTTC).

Turismo de naturaleza como una alternativa de desarrollo local, en condiciones de cambio climático en San Ignacio, BCS: Estudio enfocado al turismo alternativo en uno de los poblados del Municipio de Mulegé. La investigación se apega completamente a las intenciones de la presente, ya que se presenta al turismo alternativo como una alternativa de desarrollo local.

Medición del potencial turístico de aventura en Guerrero Negro B.C.S. México: Presenta un método que permite determinar el potencial para el desarrollo de actividades turísticas de aventura en Guerrero Negro, este estudio es considerado de los más específicos al no generalizar al turismo alternativo, sino que es directamente sobre una de las modalidades: el turismo de aventura.

Análisis socieconómico y perspectivas de desarrollo del aviturismo en Guerrero Negro, BCS: Determina no sólo el tipo de turismo, sino que mide al aviturismo como alternativa. El aviturismo es una de las actividades del Ecoturismo. 


\section{METODOLOGÍA}

Los periodos de tiempo corresponden de septiembre a noviembre del año 2016. Durante septiembre se delimitó la zona de estudio resultando dos municipios de la parte norte de BCS; Mulegé y Comondú (ver figura 1). Dichos municipios tienen la extensión de $32,000.37 \mathrm{~km}^{2}$ y $18,354.8 \mathrm{~km}^{2}$ respectivamente, en conjunto representan el $66.23 \%$ del territorio estatal, siendo $49.2 \%$ de Mulegé y $17.03 \%$ de Comondú (Instituto Nacional para el Federalismo y el Desarrollo Municipal [INAFED], 2016).

Los municipios, dado que su mayoría de suelo es llano, comparten características geológicas al presentar formaciones naturales similares. La diferencia orbita en que Comondú cuenta con un cordón orográfico de la Sierra La Giganta ubicada en la parte este del municipio con una elevación de 1680 metros sobre el nivel del mar (MSN) y que Mulegé presenta la Sierra San Francisco con una elevación de 1,738 MSN (INAFED, 2016). En la cuestión del clima encontramos que Mulegé presenta menor temperatura, siendo la anual máxima de 22 y $18^{\circ} \mathrm{C}$, mientras que en Comondú las temperaturas oscilan entre 31 y $29^{\circ} \mathrm{C}$ (INAFED, 2016).

De las geoformaciones podemos mencionar que ambos municipios cuentan con oasis que benefician su diversidad de flora y fauna. En Comondú existen los oasis de San Isidro y la Purísima, mientras que en Mulegé existen los oasis de Mulegé y San Ignacio. En lo referente a actividades económicas Comondú es principalmente agrícola y pecuario; Mulegé, por otro lado está dedicado a la agricultura en la parte central y a la industrial en la parte sur y norte (INAFED, 2016).

Figura 1. División de los municipios de Baja California Sur y área de estudio, en verde superior Mulegé, en verde inferior Comondú.



Fuente: INEGI (2016). 
Durante el mes de septiembre se elaboró un cuadro que permitiera visualizar los resultados obtenidos para a partir de estos proponer actividades que representen una oportunidad dentro del turismo en la región (ver cuadro 1).

Cuadro 1. Análisis de las investigaciones para determinar las actividades de turismo alternativo pertinentes.

\begin{tabular}{|c|c|c|c|}
\hline Nombre del Estudio & $\begin{array}{c}\text { Fecha de } \\
\text { investigación }\end{array}$ & $\begin{array}{l}\text { Resultado de } \\
\text { investigación }\end{array}$ & $\begin{array}{l}\text { Vertiente del } \\
\text { Turismo } \\
\text { Alternativo }\end{array}$ \\
\hline $\begin{array}{l}\text { Medición del potencial turístico de aventura } \\
\text { en Guerrero Negro B.C.S. México }\end{array}$ & 2017 & Positivo & $\begin{array}{l}\text { Turismo de } \\
\text { aventura }\end{array}$ \\
\hline $\begin{array}{l}\text { Evaluación del potencial turístico rural como } \\
\text { línea de acción para el desarrollo regional } \\
\text { sustentable en los oasis de San Isidro y La } \\
\text { Purísima, Baja California Sur México }\end{array}$ & 2016 & Positivo & Turismo rural \\
\hline $\begin{array}{l}\text { Desarrollo de una metodología determinar el } \\
\text { potencial del turismo rural en oasis } \\
\text { sudcalifornianos de México }\end{array}$ & 2015 & Positivo & $\begin{array}{l}\text { Turismo } \\
\text { Rural/Ecoturis } \\
\text { mo }\end{array}$ \\
\hline $\begin{array}{l}\text { Turismo de naturaleza como una alternativa } \\
\text { de desarrollo local, en condiciones de cambio } \\
\text { climático en San Ignacio, B. C. S, México }\end{array}$ & 2014 & Positivo & Ecoturismo \\
\hline $\begin{array}{l}\text { Análisis socieconómico y perspectivas de } \\
\text { desarrollo del aviturismo en Guerrero Negro, } \\
\text { Baja California Sur, México }\end{array}$ & 2012 & Positivo & Ecoturismo \\
\hline
\end{tabular}

Fuente: elaboración propia.

Durante el mes de octubre se dedicó el tiempo a la revisión y análisis de los estudios para determinar las actividades potenciales.

\section{RESULTADOS}

En el cuadro 2 encontramos que los resultados de las investigaciones son positivos, lo que permite entender que el norte de BCS no sólo tiene dicha potencialidad, sino que los estudios nos presentan oportunidades con base en datos de primera fuente. También se observa una tendencia a desarrollar investigaciones de enfoque alternativo, ya que los documentos analizados se remontan a los últimos 4 años, esto puede ser traducido como una tendencia de las vocaciones que los municipios de Mulegé y Comondú encuentran en lo referente al turismo alternativo. 
Cuadro 2. Resultados del análisis de estudio para complementar con el estudio de campo. Se presentan las actividades potenciales con base en el análisis de los documentos.

$\begin{array}{lcccc}\text { Nombre del Estudio } & \begin{array}{c}\text { Fecha de } \\ \text { investigación }\end{array} & \begin{array}{c}\text { Resultado de } \\ \text { investigación }\end{array} & \begin{array}{c}\text { Vertiente } \\ \text { del Turismo } \\ \text { Alternativo }\end{array} & \begin{array}{c}\text { Actividades } \\ \text { propuestas }\end{array}\end{array}$

\begin{tabular}{l}
$\begin{array}{l}\text { Medición del potencial } \\
\text { turístico de aventura en } \\
\text { Guerrero Negro B.C.S. México }\end{array}$ \\
\hline
\end{tabular}

Evaluación del potencial turístico rural como línea de acción para el desarrollo regional sustentable en los oasis de San Isidro y La Purísima, Baja California Sur México

\begin{tabular}{|c|c|c|c|}
\hline 2016 & Positivo & $\begin{array}{c}\text { Turismo } \\
\text { rural }\end{array}$ & $\begin{array}{l}\text { Talleres artesanales, } \\
\text { agroturismo, talleres } \\
\text { gastronómicos } \\
\text { fotografía rural }\end{array}$ \\
\hline
\end{tabular}

Desarrollo de una metodología determinar el potencial del turismo rural en oasis sudcalifornianos de México
2015

Positivo

Turismo

Rural/Ecotur

ismo

Talleres artesanales, agroturismo, fotografía rural, observación de ecosistemas, flora $y$ fauna, senderismo interpretativo, observación de geología, observación de la naturaleza

Turismo de naturaleza como una alternativa de desarrollo local, en condiciones de cambio climático en San Ignacio, B. C. S, México

Observación de naturaleza, safari fotográfico,

Senderismo interpretativo, Observación de geología

Análisis socieconómico y perspectivas de desarrollo del aviturismo en Guerrero Negro, Baja California Sur, México
Observación de fauna, ecosistemas, rescate de flora y fauna, Observación de naturaleza.

Fuente: elaboración propia.

Son entonces 16 actividades que representan oportunidades para ser desarrolladas en el área delimitada, sobresaliendo observación de ecosistemas, observación de flora y fauna, fotografía rural, agroturismo y talleres artesanales. De acuerdo a los resultados, el municipio de Comondú cuenta con 10 posibles actividades, 4 de ellas exclusivas y 6 compartidas con Mulegé, para caso de éste último cuenta con 12. Cabe mencionar que las actividades de ecoturismo son las más compartidas. En lo concerniente a los estudios del Mulegé se analizan 3, dos enfocados a la modalidad de ecoturismo y un tercero al de aventura. Para el caso de Comondú también manejan dos modalidades: rural y ecoturismo. 
Cuadro 3. Lista de actividades resultantes y municipios en los que representa una oportunidad para el turismo alternativo.

Municipio en el cual representa una oportunidad

Actividades Resultantes

Comondú Mulegé

\begin{tabular}{|c|c|c|}
\hline \multicolumn{3}{|l|}{ MODALIDAD DE ECOTURISMO } \\
\hline Observación de ecosistemas & $x$ & $x$ \\
\hline Observación de flora y fauna & $x$ & $x$ \\
\hline Observación de la naturaleza & $x$ & $x$ \\
\hline Senderismo interpretativo & $x$ & $x$ \\
\hline Observación de geología & $x$ & $X$ \\
\hline Safari fotográfico & $x$ & $x$ \\
\hline Rescate de Flora y Fauna & & $x$ \\
\hline \multicolumn{3}{|l|}{$\begin{array}{c}\text { MODALIDAD DE TURISMO DE } \\
\text { AVENTURA }\end{array}$} \\
\hline Pesca recreativa & & $x$ \\
\hline Caminata & & $x$ \\
\hline Buceo libre & & $x$ \\
\hline Bicicleta & & $x$ \\
\hline Kayak & & $x$ \\
\hline \multicolumn{3}{|l|}{ MODALIDAD DE TURISMO RURAL } \\
\hline Talleres artesanales & $x$ & \\
\hline Agroturismo & $x$ & \\
\hline Talleres gastronómicos & $x$ & \\
\hline Fotografía Rural & $x$ & \\
\hline TOTAL & 10 & 12 \\
\hline
\end{tabular}

Fuente: elaboración propia.

Es de destacar que sobresalen las actividades de ecoturismo, siendo 7 en total, 6 de ellas representan una oportunidad en ambos municipios, pues de acuerdo con Villavicencio (2014), la importancia del Oasis de San Ignacio en Mulegé se debe al resultado de la presencia de manantiales, además de su flora y fauna para la observación.

En Comondú, en el rubro del ecoturismo, Ruiz (2016) concluye que las riquezas naturales representan un valor especial por los oasis que benefician al entorno natural dotándolas de escenarios únicos con aspectos como flora, fauna, geomorfología, clima y agua.

La actividad de ecoturismo que es única para Mulegé es la de rescate de flora y fauna, debido a la existencia de la Reserva de la Biósfera de Vizcaíno (REBIVI), una Área Natural Protegida (ANP) que busca preservar los recursos naturales existentes, caso contrario a Comondú que carece de un plan de manejo por una ANP. 
Para juicio del turismo de aventura, se encontraron 5 actividades que son únicas para el municipio de Mulegé, específicamente en Guerrero Negro como lo marca el estudio de los autores. Los resultados para esta modalidad son 3 actividades por agua y 2 por tierra, ya que el sitio en mención se caracteriza por terrenos llanos para la caminata y bicicleta, por parte del agua, se cuenta con la presencia de una laguna que facilita el desarrollo de actividades como kayak, buceo libre y pesca recreativa.

Finalmente, en el aspecto del turismo rural como lo menciona Ruiz (2015) "el Turismo Rural es una actividad con alta probabilidad de ser realizado en los oasis sudcalifornianos", se muestra a Comondú como el más basto en recursos naturales dentro de sus Oasis, y como lo complementan Arce y Ruiz (2016) "el oasis de San Isidro como el de La Purísima tiene una similitud en el potencial para el desarrollo de turismo rural; es decir independientemente la época del año el agua abunda lo cual es indispensable para el desarrollo de cualquier actividad en el medio rural".

\section{CONCLUSIONES}

Como datos únicos esta investigación aporta que, si bien el norte del estado de BCS carece de infraestructura, esto le permite dedicarse a una modalidad distinta a las actividades desarrolladas en el sur. También se resalta la diversidad de oferta turística para llevar a cabo, teniendo la ventaja de que son realizadas en contacto directo con la naturaleza y donde la infraestructura tiende a pasar a segundo término. Es deber de los prestadores de servicios el aprovechar las ventajas competitivas que tiene el norte del estado en comparación con la parte sur, donde el turismo se dirige al de sol y playa. En el caso de la zona estudiada, la vocación debe dirigirse hacia una que aproveche los recursos existentes.

Se considera apropiado que la vocación turística de la zona estudiada ese oriente a actividades de turismo alternativo. Esta propuesta representa una oportunidad al ser un atractivo diferente dentro del estado y dirigido a las tendencias actuales de los nuevos turistas al ser llevadas en el medio natural. Apegándonos a García (2016), la demanda de experiencias que permiten que el turista tenga un contacto directo con la naturaleza ha tenido un significativo crecimiento. En este orden de ideas, el desarrollar la modalidad propuesta no sólo se aprovecha los recursos naturales, sino que se refiere a una actividad que se encuentra en crecimiento referente al posicionamiento dentro del mercado turístico.

En función al cuidado y preservación ecológica debemos recordar que, como lo dice Holden, "El turismo puede conservar o destruir la naturaleza" (Holden, 2016). Sin embargo, el turismo alternativo tiende a buscar la preservación de los recursos naturales con base en la apreciación y disfrute de los mismos (SECTUR, 2004). Por otro lado, García (2016) afirma que actualmente los turistas tienden a ser más conscientes sobre la necesidad de preservar recursos naturales de los destinos que visitan, es decir, el turismo alternativo comienza 
ganar importancia no solo como generador de divisas, sino como creador de conciencia ambiental dentro de los turistas.

Para las empresas turísticas se presenta una clara oportunidad de diversificar la oferta en el norte del estado. Como lo comenta Holden (2016), los beneficios de las empresas basadas en el turismo ayudan a la seguridad económica y beneficios deseados por los gobiernos, lo que nos hace coincidir con la tendencia que se desea para el estado de Baja California Sur.

Además de lo mencionado, el aprovechamiento de los recursos naturales en la zona analizada sería detonante para el sector público y para el privado, que como lo comenta Holden (2016), los beneficios de las empresas basadas en el turismo ayudan a la seguridad económica y beneficios deseados por los gobiernos (Holden, 2016), lo que nos hace coincidir con la tendencia que se desea para el estado de Baja California Sur. Como señala Cruz (2014), con este tipo de investigaciones "se contribuye propiciar una mayor difusión al exterior de los destinos turísticos de México, mejorar la conectividad aérea de centros turísticos, incentivar una mayor inversión de empresarios hoteleros, generar una mayor diversidad de servicios turísticos y generar instrumentos de reglamentación que cuiden los aspectos de sustentabilidad de los recursos naturales" (Cruz, et. al. 2014).

En este sentido, la importancia que encontramos al turismo alternativo dentro del norte de BCS se ve sustentada en distintos puntos básicos. Primeramente, el aprovechamiento de sus recursos naturales frente a la necesidad de crear su propia identidad turística al no ser beneficiado por la actividad desarrollada en el sur del estado. Además, si bien es cierto, la zona de estudio necesita enfocarse a ofertar actividades que se encuentren dentro del interés de los turistas actuales. Por ello, es de destacar que el turismo en juicio está considerado como uno de las modalidades que se ha logrado posicionar en poco tiempo como de las más representativas en función de demanda y por ende, de divisas, mismas que beneficiarían directamente en el área delimitada.

Cabe mencionar, que la importancia ecológica juega un papel prioritario dentro del turismo alternativo, puesto que las actividades han de ser desarrolladas en el medio natural, es éste considerado como materia prima, por lo que resulta necesario el conservar el recurso que permite el desarrollo de la actividad. No obstante, el turismo alternativo busca sembrar en los visitantes una conciencia de la importancia ecológica del ambiente natural, por lo que podemos entender que, además de ser generador de divisas, es él mismo quien ayuda a mantener los escenarios en condiciones de seguir siendo preservados y aprovechados.

BCS puede ser un fuerte generador de divisas en turismo debido a que en la parte sur el sector servicios se encuentra desarrollado, entonces, si el norte del territorio se enfoca en las actividades turísticas alternativas no sólo tendrá una diversificación de oferta, sino que todo el estado sería beneficiado. 


\section{REFERENCIAS BIBLIOGRÁFICAS}

Arce Meza, Javier Alberto; Ruiz Ceseña, Francisco Isaías. "Evaluación del potencial turístico rural como línea de acción para el desarrollo regional sustentable en los oasis de San Isidro y La Purísima, Baja California Sur, México". Revista Global de Negocio [en línea], 2016, Vol. 4, (No. 7). pp. 51-41 [Consulta: 16 octubre 2016]. ISSN 2328-4668. Disponible en: http://www.theibfr.com/ARCHIVE/RGN-V4N7-2016.pdf\#page=43

Bertoni, Marcela. "Recursos naturales en nodos turísticos". Aportes y transferencias [en línea], 2005, Vol. 9 (No. 2), pp.95-111. [Consulta: 23 julio 2017]. ISSN: 0329-2045. Disponible en: < http://www.redalyc.org/pdf/276/27690206.pdf>.

Boullón, Roberto C. Planificación del espacio turístico. Cuarta Edición. México, DF.: Trillas 2006. ISBN: 968-24-7562-7

Covarrubias Ramírez, Rafael. Evaluación del potencial en municipios turísticos a través de metodologías participativas. El caso de los municipios de la zona norte de Colima, México. [libro electrónico]. EUMED.NET, 2015. [Consulta: 23 julio de 2017]. Disponible en < http://www.eumed.net/libros-gratis/2015/1433/\#indice>.

Cruz Chávez, Gustavo Rodolfo; Hernández Trejo, Víctor Ángel; Avilés Polanco, Gerzaín; Juárez Mancilla, Judith. "Centros Integralmente Planeados para el turismo y crecimiento económico a largo plazo en México. Evidencia empírica para Los Cabos". En: Estrategias de desarrollo Turístico y Agroindrustrial en los estados del Pacífico Mexicano. La Paz, Baja California Sur, México: Cuadernos Universitarios UABCS, 2015. pp. 33-66.

Cruz Chávez, Plácido Roberto; Juárez Mancilla, Judith; Urciaga García, José; Ruiz Ceseña, Francisco Isaías. "Perspectivas Del Turismo: Caso México". Global Conference on Business and Finance Proceedings [en línea], 2014, Vol. 8 (No. 1), pp. 714-721. [Consulta: 24 octubre 2016]. ISSN 1941-9589. Disponible en: <http://s3.amazonaws.com/academia.edu.documents/46495232/ISSN-1941-9589-V8N1-

2013.pdf?AWSAccessKeyId=AKIAIWOWYYGZ2Y53UL3A\&Expires=1497042675\&Signatu re=yXk3cCfYQn3Mcust5z\%2Fkl8wCHJM\%3D\& response-content-

disposition=inline\%3B\%20filename\%3DCREATIVE_INDUSTRY_AN_OVERVIEW_OF_THE _STA.pdf\#page $=735>$.

Elizondo Torres, Rodolfo; Cárdenas Sosa, Carolina; Solís Trejo, Ma. Teresa. Elementos para Evaluar el Impacto Económico, Social y Ambiental del Turismo de Naturaleza en México [libro electrónico]. México, D.F. 2007. [Consulta: 23 julio de 2017]. Disponible en: < http://ictur.sectur.gob.mx/pdf/estudioseinvestigacion/sustentabilidad/METODOLOGIA _SUSTENTABILIDAD_1d3.pdf >.

García López, Raúl. Principales tendencias del turismo en 2016 [en línea]. Raúl García López, 17 de enero de 2016. [Consulta: 23 de octubre de 2016]. Disponible en < http://www.aprendedeturismo.org/principales-tendencias-del-turismo-en-2016/>.

Goeldner, Charles R.; Ritchie Brent. Tourism: Principles, Practices, Philosophies. 12va edición. Estados Unidos: John Wiley \& Sons Inc., 2011. ISBN: 978-1118071779 
Hernández Álvarez, Norma Adriana. Análisis Socieconómico Y Perspectivas De Desarrollo Del Aviturismo En Guerrero Negro, Baja California Sur, México [Tesis]. La Paz, Baja California Sur: 2012. [Consulta: 18 octubre de 2016]. Disponible en <http://biblio.uabcs.mx/tesis/TE2810.pdf>.

Holden, Andrew. Environment and tourism. [Libro electrónico] 3era edición. New York, NY: Routledge. Milton Park, Abingdon, Oxon 2016. [Consulta: 02 noviembre 2016]. Disponible en https://books.google.com.mx/books?hl=es\&lr=\&id=1WQRDAAAQBAJ\&oi=fnd\&pg=PP1 \&dq=holden+environment+and+tourism\&ots=sxjusxUvp6\&sig=1NHvL8j6eUNLiX5R9Jtd1JZ3_4\#v=onepage\&q=holden\%20environment\%20and\%20tourism\&f $=$ false>

Ibáñez Pérez, Reyna María; Cruz Chávez, Plácido Roberto; Juárez Mancilla, Judith. "Perfil y satisfacción del visitante del destino: Los Cabos, Baja California Sur". Opción [en línea], 2016, Vol. 32(No. 13), pp.1041-1068. [Consulta: 06 octubre 2016]. ISSN 1012-1587. Disponible en: <http://www.redalyc.org/pdf/310/31048483049.pdf>.

Instituto Mexicano para la Competitividad. Nueva Política Turística para recuperar la Competitividad del Sector y detonar el Desarrollo Regional [en línea]. 2013 [Consulta: 16 octubre 2016] Disponible en: <http://imco.org.mx/wpcontent/uploads/2013/10/Turismo2013_Completo.pdf>

Informador. Sube PIB trimestral en turismo en México [en línea]. Informador: 2015. [Consulta: 22 de octubre de 2016]. Disponible en: $<$ http://www.informador.com.mx/economia/2015/608117/6/sube-pib-trimestral-enturismo-en-mexico.htm>

Instituto Nacional de Ecología; Secretaría del Medio Ambiente y los Recursos Naturales. Programa de Manejo de Reserva de la Biósfera "El Vizcaino" [libro electrónico], México DF: Dirección Ejecutiva de Participación Social, 2000. [Consulta: 22 de octubre de 2016]. Disponible en: <http://www.conanp.gob.mx/datos_abiertos/DGCD/49.pdf>.

Instituto Nacional para el Federalismo y el Desarrollo Municipal. Enciclopedia de los Municipios y Delegaciones de México: Comondú [en línea]. 2016 [Consulta: 02 octubre 2016] Disponible en: <http://www.inafed.gob.mx/work/enciclopedia/EMM03bajacaliforniasur/municipios/ 03001a.html>.

Instituto Nacional para el Federalismo y el Desarrollo Municipal. Enciclopedia de los Municipios y Delegaciones de México: Mulegé [en línea]. 2016 [Consulta: 02 octubre 2016] Disponible en: http://siglo.inafed.gob.mx/enciclopedia/EMM03bajacaliforniasur/municipios/03002a. html>

Landa Pérez, Jaime Enrique. El turismo religioso y el desarrollo turístico de la parroquia totoras cantón Ambato provincia de Tungurahua. [Tesis]. Ambato, Ecuador: Universidad Técnica de Ambato, 2015. [Consulta: 24 de julio de 2017]. Disponible en < http://repo.uta.edu.ec/browse?type=author\&value=Landa+P\%C3\%A9rez\%2C+Jaime+E nrique $>$.

López, Jesarela; González, Carmen. Diagnóstico del sector turismo en México. México: Fundación Preciado. 2012. 
Mendoza Davis, Carlos. No es ni siquiera un impuesto, es un aprovechamiento [en línea]. La Paz, Baja California Sur. 2016. [Consulta: 02 de octubre de 2016]. Disponible en <http://peninsulardigital.com/extra/no-siquiera-impuesto-aprovechamiento/208301>.

Organización Mundial del Turismo. Las llegadas de turistas internacionales suben en 2015 [en línea] Organización Mundial del Turismo, 2015. [Consulta: 04 de octubre de 2016]. Disponible en: <http://media.unwto.org/es/press-release/2016-01-18/las-llegadas-deturistas-internacionales-suben-en-2015-un-4-hasta-el-record>.

Ortiz Zambrano, Aurelio. Genera Los Cabos el 65 \% del PIB estatal [en línea]. La Paz, Baja California Sur. 2013. [Consulta: 02 octubre de 2016]. Disponible en $<$ http://peninsulardigital.com/municipios/loscabos/genera-los-cabos-el-65-del-pibestatal/122174>.

Picasso Salazar, Emmanuel; Ruiz Ceseña, Francisco Isaías. "Medición del potencial turístico de aventura en Guerrero Negro, BCS, México". Revista Global de Negocios [en línea], 2017, Vol. 5 (No. 5), pp. 63-73. [Consulta: 19 de octubre de 2016]. ISSN 2328-4668. Disponible en: <https://papers.ssrn.com/sol3/papers.cfm?abstract_id=2916369>.

Quesada Castro, Renato. Elementos del turismo. Cuarta reimpresión corregida. San José, Costa Rica: Editorial Universidad Estatal a Distancia, 2007. ISBN: 9968-31-057-3.

Reachi Lugo, Rubén. El sector turístico de Baja California Sur en franco crecimiento y consolidación [en línea]. La Paz, Baja California Sur. [Consulta: 06 octubre de 2016]. Disponible en <http://descubrebajasur.com/?p=1636>.

Ruiz Ceseña, Francisco Isaías. "Desarrollo De Una Metodología Para Determinar El Potencial Del Turísmo Rural En Oasis Sudcalifornianos De México". Revista Global de Negocios [en línea], 2016, Vol. 4 (No. 8), pp. 23-34. [Consulta: 19 de octubre de 2016]. ISSN 23284668. Disponible en: <https://papers.ssrn.com/sol3/papers.cfm?abstract_id=2802279>

Secretaría de Turismo. Turismo Alternativo, una nueva manera de hacer turismo [en línea]. México, DF: Secretaría de Turismo, 2004. [Consulta: 15 octubre de 2016]. Disponible en: $\quad<$ http://www.entornoturistico.com/wp-content/uploads/2017/05/TurismoAlternativo-una-nueva-forma-de-hacer-turismo.pdf>.

Sistema Integral de Información de Mercados Turísticos. Reporte de actividad hotelera por estado. [en línea]. 2016. Disponible en <http://201.159.142.19/Cognos/cgi bin/cognos.cgi?b_action=cognosViewer\&ui.action=run\&ui.object=\%2fcontent $\% 2$ fpack age\%5b\%40name\%3d\%27siimt_master\%27\%5d\%2freport\%5b\%40name\%3d\%27ACTIV IDAD_HOTELERA_ESTADO_ORDEN\%27\%5d\&ui.name=ACTIVIDAD_HOTELERA_ESTADO _ORDEN\&run.O>.

Tisdell, Clem. "Socieconomic causes of loss of animal genetic diversity: analysis and assessment". Ecological Economics [en línea], 2003, Vol. 45 (No.3), pp. 365-376. [Consulta: 24 julio de 2017]. Disponible en < http://www.sciencedirect.com/science/article/pii/S0921800903000910>.

Villavicencio Liera, Belén. Turismo De Naturaleza Como Una Alternativa De Desarrollo Local, En Condiciones De Cambio Climático En San Ignacio, B. C. S, México [Tesis]. La Paz, Baja California Sur: 2014. [Consulta: 22 octubre de 2016]. Disponible en: <http://biblio.uabcs.mx/tesis/te3178.pdf>.

Zamorano Casal, Francisco Manuel. Turismo Alternativo, Servicios Turísticos Diferenciados. México, D.F.: Trillas, 2008. ISBN: $9682480108,9789682480102$. 